\title{
Causal beliefs about intellectual disability and schizophrenia and their relationship with awareness of the condition and social distance
}

\author{
Katrina Scior \& Adrian Furnham
}

Research Department of Clinical Educational \& Health Psychology, University College London, Gower Street, London WC1E 6BT

Corresponding author:

K Scior, k.scior@ucl.ac.uk

Running title: Causal beliefs, awareness and social distance

Keywords: intellectual disability, schizophrenia, mental disorders, social stigma, social distance, social perception 


\begin{abstract}
Evidence on mental illness stigma abounds yet little is known about public perceptions of intellectual disability. This study examined causal beliefs about intellectual disability and schizophrenia and how these relate to awareness of the condition and social distance. UK lay people aged $16+(\mathrm{N}=1752)$, in response to vignettes depicting intellectual disability and schizophrenia, noted their interpretation of the difficulties, and rated their agreement with 22 causal and four social distance items. They were most likely to endorse environmental causes for intellectual disability, and biomedical factors, trauma and early disadvantage for schizophrenia. Accurate identification of both vignettes was associated with stronger endorsement of biomedical causes, alongside weaker endorsement of adversity, environmental and supernatural causes. Biomedical causal beliefs and social distance were negatively correlated for intellectual disability, but not for schizophrenia. Causal beliefs mediated the relationship between identification of the condition and social distance for both conditions. While all four types of causal beliefs acted as mediators for intellectual disability, for schizophrenia only supernatural causal beliefs did. Educating the public and promoting certain causal beliefs may be of benefit in tackling intellectual disability stigma, but for schizophrenia, other than tackling supernatural attributions, may be of little benefit in reducing stigma.
\end{abstract}

\title{
1. Introduction
}

Lay causal beliefs about mental illness have found a lot of attention in the empirical literature. There has been much debate, particularly in relation to schizophrenia, how different causal beliefs or conceptualisations affect social distance, as measure of stigma (Angermeyer and Matschinger, 2005; Schomerus et al., 2013). This question has important implications for anti-stigma interventions. Causal attributions associated with higher levels of 
stigma should be discredited, whereas those associated with lower levels of stigma are obvious ones to reinforce. The most hotly contested question is whether promoting biological explanations has a positive effect on stigma or the reverse (Angermeyer et al., 2011; Corrigan and Watson, 2004; Jorm and Griffiths, 2008; Jorm and Oh, 2009; Kvaale et al., 2013; Read et al., 2006; Speerforck et al., 2014). Emphasising biological factors and parallels between physical and mental illness can be expected to reduce blame from the individual and hence stigma in line with attribution theory, as difficulties are attributed to factors outside the individual's control (Weiner, 1985). Conversely if difficulties are attributed to causes within the individual's control, attribution theory predicts that others are less willing to interact with a person.

However, the likening of mental illness to a 'brain disease' may unintentionally increase stigma by enhancing perceptions of unpredictability and dangerousness (Angermeyer et al., 2011; Read et al., 2006) and by making the person seem 'defective' and 'almost a different species' (Phelan, 2002). Evidence suggests that biological causal explanations do not necessarily have a positive effect on levels of stigma (Dietrich et al., 2004). The authors argued that biological causes and those that a person can influence themselves may be associated with a perceived lack of control, such as loss of cognitive control in the case of brain damage or loss of personal control in the case of laziness attributed to a "weak character". Hence both attributions may lead others to view the person as dangerous and unpredictable.

The evidence is mostly derived from vignette based studies, and in some cases by inviting lay people to respond directly to diagnostic labels. One important question to address in using diagnostically unlabelled vignettes is whether the causal beliefs of those who identify the symptoms presented as signs of the respective condition differ from the causal beliefs of those who interpret the behaviours presented differently. The present study 
attempted to do so, while also linking these processes to stigma. Understanding how lay causal beliefs relate to awareness of intellectual disability and schizophrenia and to stigma is important for a number of reasons. Evidence on the public's causal beliefs and stigma can inform public education efforts and identify what messages are most helpful. In addition, the integration of all three aspects in empirical inquiries allows us to identify the respective contributions of awareness and different causal beliefs to social distance and thus what targets to choose to have the greatest effect on stigma.

\subsection{Lay beliefs about intellectual disability}

In contrast to the burgeoning mental health literature, evidence on the general public's conceptualisations about intellectual disability is thin on the ground. A review identified only five studies during the period 1990 to 2010 that looked at lay people's beliefs about the causes of intellectual disabilities (Scior, 2011). Only two of these examined the relationship between causal beliefs about intellectual disability and stigma. In a US-based study, intellectual disability due to genetics was perceived most positively, while "self-inflicted" disability, in this case due to drinking cleaning fluid in childhood, was viewed most negatively (Panek and Jungers, 2008). In a study conducted in Ethiopia, supernatural retribution was deemed one likely cause of intellectual disability that was in turn associated with more negative attitudes (Mulatu, 1999). Studies in India and Tanzania identified lay causal beliefs, including a belief that intellectual disability may be due to 'god's will', parents' actions and transgressions of social or religious rules or witchcraft (Kisanji, 1995; Madhavan et al., 1990). Only 4\% of lay people in India saw prenatal complications or heredity as likely causes (Madhavan et al., 1990). Significant misconceptions about the causes of Down Syndrome among the Australian public were identified by Gilmore et al. (2003), including $26 \%$ of respondents believing the condition to be caused by parental lifestyle or problems during birth. While these studies provide some useful pointers, they are 
mostly limited by small sample sizes and provide limited evidence on the effect of different causal beliefs on stigma.

A study of Pakistani parents of children with intellectual disabilities found that all parents made reference to theological explanations as to why they had a child with a disability, but most also gave biomedical or other explanations (Croot et al., 2008). Parents often gave theological explanations initially, but resorted to biomedical discourse when facing negative or unhelpful ideas. Their findings are in line with Hatton et al. (2003), who noted that parents who have a good understanding of the medical explanation for their child's disability appear to use this to refute unhelpful beliefs about the causes of their child's disability among their extended family and expectations of a 'cure'. Thus the idea that biomedical explanations can lower stigma is certainly present within the intellectual disability literature, but at present is poorly articulated and not empirically tested.

\subsection{Aims of the Study}

This study set out to investigate the relationship between lay knowledge, causal beliefs and social distance in relation to intellectual disability and schizophrenia. The research questions were: 1) what beliefs about the likely causes of typical symptoms of (mild) intellectual disability and schizophrenia are prevalent in the UK?; 2) what effect does awareness of intellectual disability/schizophrenia, as evidenced by the ability (or lack thereof) to recognise symptoms of the respective condition in a diagnostically unlabelled vignette, have on causal beliefs and social distance? In particular, do people who recognise the condition attribute more importance to biomedical factors, and less to psychosocial and supernatural factors?; and 3) what is the association between causal beliefs and social distance? Finally, we hypothesised that the relationship between knowledge of the respective condition and social distance is mediated by participants' causal beliefs. These processes 
were examined in relation to intellectual disability and schizophrenia to ascertain whether they are disorder specific or more generic.

\section{Methods}

\subsection{Participants}

A cross-sectional survey involving a convenience sample of 1752 adult UK residents was conducted. The majority were female and their mean age was 25.4 years (range 16 to 79 years). All participants were either UK nationals or had been resident in the UK for at least 3 years. The sample was very ethnically mixed. Prior contact with someone with mental health problems was reported by $46.4 \%$, and prior contact with someone with intellectual disabilities by $32.6 \%$. Demographic data are provided in Table 1 .

[Table 1 about here]

\subsection{Procedure}

Participants were recruited via email to the social contacts of the authors and junior researchers involved in the project, the social networking site Facebook, and advertisements on internet forums. Facebook recruitment comprised of the recruitment email being posted on open public online groups with a request to invite others to the group. Advertisements containing information about the study and a link to the on-line survey site were placed on on-line discussion forums. In addition, participants were asked to forward the recruitment email to their distribution lists. The recruitment email and advertisements asked potential participants to complete a brief questionnaire on their views of "personal difficulties in others", and gave the option of entering a prize draw for retail vouchers designed to incentivise recruitment. Of those who accessed the survey $(\mathrm{N}=3363), 52.1 \%$ completed it fully or had less than $5 \%$ of missing data and were included in the analyses. Ethical approval for the study was granted by the authors' institution.

\subsection{Measures}


Participants were presented with two unlabelled vignettes of a male in his 20 s. The first depicted an individual who met diagnostic criteria for a (mild) intellectual disability, the other for schizophrenia (World Health Organisation, 1990), see Appendix. Respondents were asked "what would you say is going on with X?". They then indicated their agreement with 22 possible causes of the person's difficulties, on a 7-point Likert scale $(1=$ strongly disagree to $7=$ strongly agree) (see Scior and Furnham, 2011, for development of the measure). Participants also rated their willingness to have social contact with someone like the person in the vignette by responding to four statements about social contact in situations of increasing intimacy (live next door, spend an evening socialising, make friends, marry into family), taken from Link, Phelan, Bresnahan, Stueve and Pescosolido (1999), using the same 7-point Likert scale.

Responses to the 22 causal items were analysed under four subscales: biomedical, adversity, environmental, and supernatural causes (Scior and Furnham, 2011). The adversity and environment subscales encompassed a range of psychosocial explanations. For each subscale a mean score of the constituent items was calculated. Higher scores indicate stronger endorsement of the respective cause. A mean score of the reversed social distance items was calculated; higher scores indicate greater social distance. The internal consistency of the causal subscales was very good for both vignettes. Cronbach alphas for the intellectual disability vignette were: biomedical $\alpha=0.85$; adversity $\alpha=0.81$; environment $\alpha=0.79$; and supernatural $\alpha=0.78$. For the schizophrenia vignette the internal reliability of the causal subscales was also good: biomedical $\alpha=0.80$; adversity $\alpha=0.81$; environment $\alpha=0.84$; and supernatural $\alpha=0.81$. Inter-item correlations were between 0.17 and 0.63 . Participants also provided detailed socio-demographic information.

\subsection{Statistical analysis}


The data were analysed using SPSS version 21. Responses to the open ended recognition question were coded into 12 broad categories for each vignette. If multiple labels for the presentation were suggested, any response that included a reference to the correct diagnosis was counted. All responses that failed to name the correct diagnosis were coded into 11 other categories, such as whether the respondent attributed the presentation to a specific learning difficulty or autism, another specified type of mental illness, or mental illness in general, a character defect in the individual depicted, more typical adolescent disruption, or the person's parents' or teachers' failings.

Exploration of the data indicated that three of the four causal belief subscales were normally distributed. However the supernatural subscale showed large positive skewness for both intellectual disability and schizophrenia; most participants disagreed with such causes, regardless of condition. Log transformation of this subscale resolved this problem. The internal consistency of the causal subscales was very good for both vignettes across the entire sample. Cronbach alphas for the intellectual disability vignette were biomedical $\alpha=0.85$, adversity $\alpha=0.81$, environment $\alpha=0.79$, and supernatural $\alpha=0.78$. For the schizophrenia vignette the internal reliability of the causal subscales across the entire sample was also good, with biomedical $\alpha=0.80$, adversity $\alpha=0.81$, environment $\alpha=0.84$, and supernatural $\alpha=0.81$. Inter-item correlations were between 0.17 and 0.63 .

Paired samples $t$ tests were used to compare endorsement of the four causal beliefs between the two vignettes. The effect of explanation given for the vignette presentation on causal beliefs was examined using ANOVAs and post hoc analyses. To examine what associations exist between different causal beliefs and social distance correlations were calculated. To test the hypothesis that the relationship between knowledge about the respective condition and social distance is mediated by participants' causal beliefs, mediation 
analyses were performed. Missing data were handled through pairwise deletion. Effect sizes are reported throughout as Cohen's $d$.

\section{Results}

\subsection{Causal Beliefs}

For the intellectual disability vignette, a history of poor schooling, lack of daytime occupation, overly lenient parenting and brain abnormality received the strongest endorsement, see Table 2. For the schizophrenia vignette, brain abnormality, genetic factors, recent trauma or bereavement were rated as most likely causes. Of the five biomedical causes, three were judged as more likely causes of the schizophrenia presentation (brain infection, genetic factors and brain abnormality), while complications at birth were deemed a more likely cause of intellectual disability. All adversity causes were deemed more likely causes of the schizophrenia presentation, while most environmental causes were judged as more likely causes of intellectual disability. Finally, spirit possession, strong religious or spiritual beliefs and retribution for parental wrongdoings were seen as less likely causes of intellectual disability, but the effect sizes were small.

[Table 2 about here]

Looking at subscale scores, participants were less likely to endorse biomedical causal explanations, $t(1478)=-6.18, p<0.001, d=0.19$, and adversity causes, $t(1478)=-9.15, p<0.001$, $d=0.18$ for intellectual disability than for schizophrenia, with small effect sizes. They were far more likely to endorse environmental explanations for intellectual disability, $t(1478)=30.21$, $p<0.001, d=0.82$. Agreement with supernatural causes did not differ between the two conditions, $t(1478)=-0.17, p=0.86$.

Participants' causal beliefs by interpretation of the respective vignette are presented in Tables 3 and 4. They were most likely to endorse environmental causes in response to the intellectual disability vignette, with adversity and biomedical causes following closely 
behind. One-way ANOVAs revealed a significant effect of interpretation of the vignette on all four causal belief subscales. To account for different sample sizes, post hoc tests were performed using Hochberg's GT2 for the adversity and supernatural causes subscales given that Levene's test was not significant, and the Games-Howell procedure for the biomedical and environmental causes subscales given that Levene's test was significant, $p<0.001$. These tests indicated that identification of the vignette as intellectual disability was associated with stronger endorsement of biomedical causes, $p<0.001, d=0.84$; alongside weaker endorsement of adversity, $p<0.01, d=0.24$; environmental causes, $p<0.001, d=0.84$; and supernatural causes, $p<0.001, d=0.54$, compared to those who failed to identify possible intellectual disability, with large effect sizes for endorsement of biomedical and environmental causes. Causal beliefs of those who identified intellectual disability and those who thought the presentation might be due to specific learning difficulties (LD) and autism (ASD) were combined in subsequent analyses as these three groups were similar in their endorsement of the four types of causes, biomedical: $p=0.27$; adversity: $p=0.44$; environment: $p=0.91$; and supernatural: $p=0.94$.

[Table 3 about here]

For the schizophrenia vignette, adversity and biomedical causes were most strongly endorsed. Environmental causes were endorsed much less than for the intellectual disability vignette, perhaps due to a perception that this presentation seemed much more serious than the intellectual disability vignette. Participants tended to disagree with supernatural causes for both vignettes. Differences in participants' causal beliefs by explanation for the schizophrenia vignette are presented in Table 4.

[Table 4 about here]

As for intellectual disability, one-way ANOVAs showed a significant effect of interpretation of the schizophrenia vignette on all four causal belief factors. Post hoc tests 
were performed using Hochberg's GT2 for the biomedical and adversity causes subscales given that Levene's test was not significant. The Games-Howell procedure was applied for the environmental and supernatural causes subscales given that Levene's test was significant at $p=0.01$ for environment and $p<0.001$ for supernatural causes. These tests showed that identification of the vignette as schizophrenia/psychosis was associated with stronger endorsement of biomedical causes, $p<0.001, d=0.67$; alongside weaker endorsement of adversity, $p<0.01, d=0.27$; environmental causes, $p<0.001, d=1.00$; and supernatural causes, $p<0.001, d=1.07$, compared to those who failed to recognise mental illness. Furthermore, identification of the vignette as schizophrenia/psychosis, rather than more general reference to other forms of mental illness, enhanced this effect, although only the results for environmental, $p<0.001, d=0.39$, and supernatural causes, $p<0.001, d=0.39$, reached significance.

Participants who attributed the behaviours in the second vignette to depression, compared to those who recognised schizophrenia, were less likely to endorse biomedical causes, $p<0.001, d=0.66$, and more likely to agree with the other three causes, adversity: $p<0.001, d=0.49$; environment: $p<0.001, d=0.88$; supernatural: $p<0.001, d=0.63$. They were as likely as participants who failed to recognise mental illness altogether to endorse biomedical causes, $p=1.00$; adversity, $p=0.25$; or environmental causes, $p=0.53$. The only difference found between these two groups was the depression group's lower endorsement of supernatural causes, $p<0.001, d=0.44$, even though this difference was much smaller than the difference in agreement with supernatural causes between the schizophrenia group and the group who failed to recognise mental illness altogether, see above. 


\subsection{Causal beliefs and social distance}

In order to identify what associations exist between different causal beliefs and social distance, regardless of participants' interpretations of the symptoms in the vignette, correlations were calculated, initially for individual causal items, see Table 5.

[Table 5 about here]

The results point to some interesting similarities and differences regarding the relationship between causal beliefs and social distance for intellectual disability and schizophrenia. Several items were associated with increased social distance for both conditions: spirit possession, punishment for own or parents' past wrongdoings, and excessive lenience on the part of parents. Of the five items constituting the supernatural scale, three were positively correlated with social distance for intellectual disability and all five for schizophrenia. There were some notable differences though for items subsumed under the biomedical subscale: virus/cerebral infection and brain abnormality were negatively correlated with social distance for intellectual disability, yet positively for schizophrenia. Genetic factors were associated with decreased social distance for intellectual disability but showed no association for schizophrenia. Notably, for schizophrenia none of the items showed a negative correlation with social distance. Overall though, the correlation coefficients for schizophrenia in particular were low (highest 0.12 ), suggesting only a weak relationship between causal beliefs and social distance.

Subsequently the relationships between causal beliefs and social distance were examined by focusing on the four subscales, see Table 6 .

[Table 6 about here]

For both intellectual disability and schizophrenia, belief in supernatural causes showed a positive correlation with social distance, although for intellectual disability this did not reach significance at the $5 \%$ level once the Bonferroni correction was applied. However, 
while belief in biomedical causes was negatively correlated with social distance for intellectual disability, no significant correlation between biomedical beliefs and social distance was observed for schizophrenia. Endorsement of environmental causes was positively correlated with social distance for intellectual disability, but not for schizophrenia. Finally, endorsement of adversity causes was not significantly correlated with social distance for either condition.

\subsection{Relationship between knowledge, causal beliefs and social distance}

To test the hypothesis that the relationship between awareness of the respective condition and social distance is mediated by participants' causal beliefs, mediation analyses were performed. Accordingly bootstrapping analyses were conducted to estimate direct and indirect effects with multiple mediators using Preacher and Hayes' (2008) script. Separate path models were generated for intellectual disability and schizophrenia to ascertain whether mediation processes are disorder specific or common to both conditions considered in this study. While it may have been desirable to adjust the analyses for co-variates, such as demographics, this was not done as this study used a within subjects design and the primary focus was on comparing the relationship between the three dependent variables across the two conditions.

For the purpose of analysis, 'intellectual disability literacy' was defined as mention of intellectual disability (or one of its synonyms) or interpretation of the behaviours as possible signs of specific learning disability (LD) or autism spectrum disorder (ASD), given that the responses of these two groups were similar on all outcomes. Results indicated that the total effect of intellectual disability literacy on social distance of $-0.79, p<0.001$ became smaller when causal belief mediators were included in the model (direct effect $=-0.42, p<0.001$ ). The total indirect effect of intellectual disability literacy on social distance through causal belief mediators was significant, $p<0.001$, with a point estimate of -0.37 and a $95 \%$ bias-corrected 
and accelerated $(\mathrm{BCa})$ bootstrap confidence interval of -0.48 to -0.27 . Therefore causal beliefs fully mediated the association between awareness of the condition and social distance for intellectual disability. The specific indirect effects of each proposed mediator showed that belief in biomedical causes, with a point estimate of -0.08 , adversity causes, with a point estimate of 0.07 , and environmental causes, with a point estimate of -0.29 were significant mediators, see Figure 1 . Overall the model explained $11 \%$ of the variance in social distance towards the individual presenting with symptoms of intellectual disability.

[Figure 1 about here]

For schizophrenia, on the basis of the comparisons between the four explanation groups, 'schizophrenia literacy' was defined as recognition of schizophrenia/psychosis, and compared to those who failed to recognise mental illness altogether. Participants who made general reference to mental illness, a different psychiatric diagnosis, or depression, were excluded from the path analysis as their causal beliefs and social distance differed from the schizophrenia group. Results indicated that the total effect of schizophrenia literacy on social distance of $-0.23, p=0.06$ became smaller when causal belief mediators were included in the model (direct effect $=0.12, p=0.42)$. The total indirect effect of schizophrenia literacy on social distance through causal belief mediators was significant, $p<0.001$, with a point estimate of -0.35 and a $95 \%$ BCa bootstrap confidence interval of -0.56 to -0.17 . Therefore, for schizophrenia causal beliefs fully mediated the association between awareness of the condition and social distance. The specific indirect effects of each proposed mediator showed that supernatural causal beliefs, with a point estimate of -0.35 were significant mediators, $p<0.001$, and the role of adversity causal beliefs, with a point estimate of .04 , approached significance, $p=0.06$. Biomedical causal beliefs, with a point estimate of 0.02 , and environmental causal beliefs, with a point estimate of -0.06 did not add to the overall model, 
see Figure 2. Overall the model explained only $5 \%$ of the variance in social distance towards the individual presenting with symptoms of schizophrenia.

In sum, for intellectual disability, biomedical, adversity and environmental, but not supernatural, causal beliefs mediated the relationship between intellectual disability literacy and social distance. In contrast, for schizophrenia only supernatural causal beliefs mediated the relationship between schizophrenia literacy and social distance; the mediating role of adversity causal beliefs approached significance. Identification of schizophrenia was associated with reduced endorsement of supernatural causes, which in turn was associated with increased social distance. As for intellectual disability, identification was associated with reduced endorsement of adversity causes, in turn associated with reduced social distance. While one might view this effect as an undesirable bi-product of increased awareness of the conditions, its effect on social distance was much less pronounced than the effect of supernatural causal beliefs.

[Figure 2 about here]

\section{Discussion}

This study set out to examine the relationships between intellectual disability and schizophrenia literacy, causal beliefs and social distance among UK adult residents, and whether these relationships are similar for both conditions or disorder specific. Participants were most likely to endorse environmental causes in response to intellectual disability, but biomedical and adversity causes in response to schizophrenia. This supports previous findings, based on a German representative population sample, that lay people tend to attribute schizophrenia to organic and other complex factors over which the person is seen to have less control, a belief in turn associated with feelings of uneasiness and fear (Angermeyer et al., 2010). The greater endorsement of biomedical causes for the schizophrenia vignette may also indicate a better understanding of the potential role of biomedical factors and 
adversity in the aetiology of mental illness, rather than intellectual disability, as a result of much greater public education and media coverage. Of note, the current intellectual disability vignette depicted someone with a mild intellectual disability - it is possible that biomedical causes would figure more prominently if the presentation was one of severe intellectual disability.

Increased intellectual disability and schizophrenia literacy were associated with increased endorsement of biomedical factors, and reduced endorsement of psychosocial and supernatural factors, in line with findings on mental illness by Schomerus et al. (2006). As predicted, recognition of schizophrenia, rather than attribution to mental illness generally, enhanced this effect. The same was not found for intellectual disability though, where no significant differences were found between those who identified intellectual disability and those who attributed the presentation to specific learning difficulties or autism spectrum disorders. This may be due to the fact that the vignette depicted someone with mild symptoms of intellectual disability, a suggestion that should be tested in further research. The differences found between those who identified schizophrenia and those who attributed the presentation to depression or mental illness generally indicate that future research should pay close attention to respondents' detailed understanding, rather than simply examining whether mental illness is identified or not.

The correlations between causal beliefs and social distance were only partly in the direction predicted. For intellectual disability, endorsement of biomedical causes was associated with reduced overall social distance, but no such association was found for schizophrenia (where two individual items were associated with increased social distance). Unexpectedly environmental causal beliefs had the strongest positive correlation with social distance for intellectual disability. Why this may be the case becomes clearer once individual items constituting the environmental subscale are considered. 'Lack of daytime occupation' 
and 'overly lenient parents' could be construed as signs of character weakness (Dietrich et al., 2004), and thus in line with attribution theory (Weiner, 1985) as more stigmatising because the person is being blamed for their difficulties. However, the distinction between causes within or outside the person's control drawn by Dietrich et al. (2004) seemed to hold neither for intellectual disability nor for schizophrenia in the current study - items such as 'overly lenient parents' could not be classified as within the person's control. Furthermore, if controllability was as strong a factor as suggested previously, one might have expected adversity items such as child abuse or recent death of a relative or friend to be associated with reduced social distance, which was neither the case for intellectual disability nor for schizophrenia. It is impossible to tell whether the differences observed might indicate that controllability attributions are perhaps less important than blame attributions, whether they may be due to changes over time or differences in the wording and contents of items, differences between lay beliefs in Germany (the focus of Dietrich et al.'s study) and the UK, or due to sampling differences (while ours was a relatively young convenience sample, Dietrich et al. used a representative population sample).

The lack of a significant correlation between biomedical causal beliefs in general and social distance in the case of schizophrenia is in line with Grausgruber et al. (2007), who used a representative population sample. More specifically though, endorsement of brain abnormality/infection as cause of the behaviours depicted in the vignette was associated with increased social distance for schizophrenia, yet with reduced social distance for intellectual disability. This could be seen to discredit attempts to destigmatise schizophrenia by teaching the public to recognise it as an illness of primarily biological aetiology, and as giving credence to arguments that such an approach in fact increases stigma.

Agreement with supernatural causes in the current study was associated with increased social distance for both intellectual disability and schizophrenia. This confirms 
concerns in the intellectual disability literature, for example, that beliefs about intellectual disability as due to punishment for past sins or wrongdoings (Hubert, 2006) and religious explanations for intellectual disability generally (Croot et al., 2008) are associated with increased stigma. However, it should be noted that such beliefs were rare in the current study, including among ethnic and religious minorities.

It is important to stress that the correlations between causal beliefs and social distance were only small for schizophrenia. Furthermore the model incorporating schizophrenia literacy and causal beliefs explained only $6 \%$ of the variance in social distance. This suggests that awareness and causal beliefs have only a weak influence on stigma associated with schizophrenia, which is perhaps much less driven by these factors than by negative stereotypes about people with schizophrenia as dangerous and potentially violent. Furthermore, the weak relationship identified between causal beliefs and social distance for schizophrenia may point to emotional reactions, which may have an important mediating role (Angermeyer et al., 2010), but were not the focus of this study. Finally, the fact that for schizophrenia eight causal items showed weak positive correlations with social distance, and none significant negative correlations, could be taken to suggest that aiming to lower the stigma associated with schizophrenia by tackling stigmatising causal beliefs may well be ineffective. In contrast, this strategy may hold more promise for intellectual disability where a much clearer relationship between causal beliefs and social distance emerged.

The hypothesis that causal beliefs mediate the relationship between awareness and social distance was confirmed for both conditions. For intellectual disability, three of the four types of causal beliefs acted as mediators. Identification of the condition on the basis of the vignette had favourable strong direct and indirect effects on social distance, the latter via the mediating effects of causal beliefs. Those who showed greater awareness of the condition were more likely to endorse biomedical causes and less likely to endorse adversity, 
environmental and supernatural causes. In turn, agreement with biomedical causes was associated with reduced social distance, while agreement with environmental and supernatural causes was associated with increased social distance.

While causal beliefs also mediated the relationship between knowledge and social distance for schizophrenia, here awareness had only a weak direct effect on social distance, and less comprehensive indirect effects than for intellectual disability. Only supernatural beliefs had a strong mediating role; identification of the condition was associated with reduced endorsement of supernatural causes, and endorsement of such causes had a strong positive effect on social distance. Identification of the presentation as possible symptoms of schizophrenia showed strong direct effects on agreement with biomedical and environmental causes, but their effects on social distance were not significant. These findings suggest that for intellectual disability, public education about the condition may have positive effects on stigma, both directly and through the mediating role of attributions. However, for schizophrenia increasing public understanding and tackling stigmatising attributions, other than supernatural beliefs, may have only limited effects on stigma. Of note, these suggestions are based on cross-sectional data and will need testing through the use of experimental designs.

Due to resource limitations, the study used convenience sampling, social media advertising and snowballing to recruit a large lay sample. Overall the sample was comparatively young; in view of this and the fact that participants were not sampled at random, caution should be exercised in generalising the findings. Internet based recruitment has numerous benefits; alongside ease of data collection and cost-effectiveness, it may reduce socially desirable responding as due to the physical distance inherent in web-surveys participants may feel less inhibited, and more willing to give honest responses (Lyons et al., 2005). However, web-surveys tend to attract younger and more educated sections of the 
population, affecting their representativeness. Another limitation concerns the fact that the order of presentation of the vignettes was kept constant rather than randomly varied - it is conceivable that this may have affected responses.

\section{Implications}

The lack of significant negative associations in this and previous studies between biomedical causal beliefs generally and social distance for schizophrenia, suggests that aiming to lower the stigma associated with schizophrenia through broad brush public education about mental illness in general and schizophrenia in particular may well be ineffective. Instead anti-stigma efforts that discourage endorsement of specific stigmatising causes, such as brain abnormality or infection, and factors beyond the scope of this study, such as negative stereotypes and emotional reactions, may show more promise. In contrast, comprehensive public education may hold promise for intellectual disability where a much clearer relationship between intellectual disability literacy, multi-faceted causal beliefs and social distance emerged.

Given that the present mediation analyses had low explanatory power, particularly for schizophrenia, future research should pay attention to complex factors beyond awareness of the condition in question and causal attributions in trying to explain stigma. 


\section{References}

Angermeyer, M.C., Holzinger, A., Matschinger, H., 2010. Emotional reactions to people with mental illness. Epidemiol. Psichiatr. Soc. 19, 26-32. Doi: 10.1017/S1121189X00001573

Angermeyer, M.C., Matschinger, H., 2005. Causal beliefs and attitudes to people with schizophrenia - trend analysis based on data from two population surveys in Germany. Br. J. Psychiatry 186, 331-334. Doi: 10.1192/bjp.186.4.331

Corrigan, P.W., Watson, A., 2004. At issue: stop the stigma: call mental illness a brain disease. Schizophr. Bull. 30, 477-479. Doi: 10.1093/oxfordjournals.schbul.a007095

Croot, E.J., Grant, G., Cooper, C.L., Mathers, N., 2008. Perceptions of the causes of childhood disability among Pakistani families living in the UK. Health Soc. Care Community 16, 606-613. Doi: 10.1111/j.1365-2524.2008.00784

Dietrich, S., Beck, M., Bujantugs, B., Kenzine, D., Matschinger, H., Angermeyer, M.C., 2004. The relationship between public causal beliefs and social distance toward mentally ill people. Aust. N. Z. J. Psychiatry 38, 348-354. Doi: 10.1111/j.1440-1614.2004.01363

Gilmore, L., Campbell, J., Cuskelly, M., 2003. Developmental expectations, personality stereotypes, and attitudes towards inclusive education: community and teacher views of Down syndrome. Intl. J. Disabil. Dev. Educ. 50, 65-76. Doi:10.1080/1034912032000053340

Grausgruber, A., Meise, U., Katschnig, H., Schöny, W., Fleischhacker, W.W., 2007. Patterns of social distance towards people suffering from schizophrenia in Austria: a comparison between the general public, relatives and mental health staff. Acta. Psychiatr. Scand. 115, 310-319. Doi: 10.1111/j.1600-0447.2006.00882

Hatton, C., Akram, Y., Robertson, J., Shah, R., Emerson, E., 2003. The disclosure process and its impact on South Asian families with a child with severe intellectual disabilities. J. Appl. Res. Intellect. Disabil. 16, 177-188. Doi: 10.1046/j.1468-3148.2003.00167

Hubert, J., 2006. Family carers' views of services for people with learning disabilities from Black and minority ethnic groups: a qualitative study of 30 families in a south London borough. Disabil. Soc. 21, 259-272. Doi: 10.1080/09687590600617451

Jorm, A.F., Griffiths, K.M., 2008. The public's stigmatizing attitudes towards people with mental disorders: how important are biomedical conceptualisations? Acta. Psychiatr. Scand. 118, 315-321.

Jorm, A.F., Oh, E., 2009. Desire for social distance from people with mental disorders: a review. Aust. N. Z. J. Psychiatry 43, 183-200. Doi: 10.1080/00048670802653349

Kisanji, J., 1995. Interface between culture and disability in the Tanzanian context: part II. Intl. J. Disabil. Dev. Educ. 42, 109-124. Doi: 10.1080/0156655950420203

Kvaale, E.P., Gottdiener, W.H., Haslam, N., 2013. Biogenetic explanations and stigma: a meta-analytic review of associations among laypeople. Soc. Sci. Med. 96, 95-103. Doi: 10.1016/j.socscimed.2013.07.017 
Link, B.G., Phelan, J.C., Bresnahan, M., Stueve, A., Pescosolido, B.A. 1999. Public conceptions of mental illness: labels, causes, dangerousness and social distance. Am. J. Pub. Health 89, 1328-1333. Doi: 10.2105/AJPH.89.9.1328

Lyons, A.C., Cude, B., Lawrence, F.C., Gutter, M., 2005. Conducting research online: challenges facing researchers in family and consumer sciences. Fam. Consum. Sci. Res. J. 33(4), 341-356. Doi: 10.1177/1077727X04274116

Madhavan, T., Menon, D.K., Kumari, R.S., Kalyan, M., 1990. Mental retardation awareness in the community. Indian J. Dis. Rehabilitation 4, 9-21. Doi: 10.1111/j.13652788.1984.tb01011

Mulatu, M.S., 1999. Perceptions of mental and physical illnesses in north-western Ethiopia: causes, treatments, and attitudes. J. Health Psychol. 4, 531-549. Doi: $10.1177 / 135910539900400407$

Panek, P.E., Jungers, M.K. 2008. Effects of age, gender, and causality on perceptions of persons with mental retardation. Res. Dev. Disabil. 29, 125-132. Doi: 10.1016/j.ridd.2007.01.002

Phelan, J.C., 2002. Genetic bases of mental illness - a cure for stigma? Trends Neurosci. 25, 430-431. Doi: 10.1016/S0166-2236(02)02209-9

Preacher, K.J., Hayes, A.F., 2008. Asympotic and resampling 'procedures for assessing and comparing indirect effects in multiple mediator models. Behav. Res. Methods 40, 879891. Doi: 10.3758/BRM.40.3.879

Read, J., Haslam, N., Sayce, L., Davies, E., 2006. Prejudice and schizophrenia: a review of the 'mental illness is an illness like any other' approach. Acta. Psychiatr. Scand. 114, 303-318. Doi: 10.1111/j.1600-0447.2006.00824

Schomerus, G., Matschinger, H., Angermeyer, M.C., 2006. Public beliefs about the causes of mental disorders revisited. Psychiatry Res. 144, 233-6. Doi: 10.1016/j.psychres. 2006.05.002

Schomerus, G., Matschinger, H., Angermeyer, M.C., 2013. Causal beliefs of the public and social acceptance of persons with mental illness: a comparative analysis of schizophrenia, depression and alcohol dependence. Psychol. Med. 44, 1-12. Doi: 10.1017/S003329171300072X

Scior, K., 2011. Public awareness, attitudes and beliefs regarding intellectual disability: a systematic review. Res. Dev. Disabil. 32, 2164-2182. Doi: 10.1016/j.ridd.2011.07.005

Scior, K., Furnham, A.F., 2011. Development and validation of the intellectual disability literacy scale for assessment of knowledge, beliefs and attitudes to intellectual disability. Res. Dev. Disabil. 32, 1530-1541. Doi: 10.1016/j.ridd.2011.01.044

Speerforck, S., Schomerus, G., Pruess, S., Angermeyer, M.C., 2014. Different biogenetic causal explanations and attitudes towards persons with major depression, schizophrenia and alcohol dependence: is the concept of a chemical imbalance beneficial? J. Affect. Disorders 168, 224-228. Doi: http://dx.doi.org/10.1016/j.jad.2014.06.013

Weiner, B., 1985. An attributional theory of achievement motivation and emotion. Psychol. Rev. 92, 548-573. Doi: 10.1037/0033-295X.92.4.548 
World Health Organisation, 1990. International Classification of Diseases and Related Health Problems 10th Revision. Geneva: World Health Organisation. 


\section{Appendix}

Intellectual Disability Vignette

James is 22 and lives at home with his parents and younger brother. He found school a struggle and left without any qualifications. He has had occasional casual jobs since. When his parents try to encourage him to make plans for his future, James has few ideas or expresses ambitions that are well out of his reach. Rather than having him at home doing nothing, his mum has been trying to teach James new skills, such as cooking a meal, but James has struggled to follow her instructions. He opened up a bank account with his parents' help, but has little idea of budgeting and, unless his parents stop him, will spend all his benefits on comics and DVDs as soon as he receives his money.

\section{Schizophrenia Vignette}

Adam is 24 and lives at home with his parents. He did fine at school, but has only had a few casual jobs since. Over recent months he has spent lots of time alone, locked in his bedroom and frequently refuses to eat with his parents or have a bath. He sometimes gets very agitated for little apparent reason and his parents have heard him talking loudly even when he's alone in his bedroom. At times they find his speech disorganised and hard to follow. When his parents encourage him to make plans for his future he says this is too dangerous. They are certain he is not taking drugs because he never sees anyone or goes anywhere. 
Table 1. Participant demographic data

\begin{tabular}{|c|c|c|}
\hline Variable & & $\mathrm{N}(\%)$ \\
\hline \multicolumn{3}{|l|}{ Gender } \\
\hline & Female & 974 (55.6) \\
\hline & Male & $704(40.2 \%)$ \\
\hline & Missing & $74(4.2 \%)$ \\
\hline \multicolumn{3}{|l|}{ Age } \\
\hline & 16 to 24 & $1163(66.4 \%)$ \\
\hline & 25 to 34 & $248(14.2 \%)$ \\
\hline & 35 to 49 & $178(10.2 \%)$ \\
\hline & 50 to 64 & $56(3.2 \%)$ \\
\hline & $65+$ & $13(0.7 \%)$ \\
\hline & Missing & $94(5.4 \%)$ \\
\hline
\end{tabular}

Education

$\begin{array}{rr}\text { To age } 16 \text { or less } & 82(4.7 \%) \\ \text { To age } 18 & 1190(67.9 \%) \\ \text { University degree } & 405(23.1 \%) \\ \text { Missing } & 75(4.3 \%)\end{array}$

Ethnicity

$\begin{array}{rr}\text { White Caucasian } & 813(46.4 \%) \\ \text { Asian } & 463(26.4) \\ \text { Black African/Caribbean } & 255(14.6) \\ \text { Other } & 131(7.5) \\ \text { Missing } & 90(5.1 \%)\end{array}$


Table 2. Endorsement of 22 causal belief items for intellectual disability and schizophrenia: Item means (standard deviations) and results of within-subjects tests

\begin{tabular}{|c|c|c|c|c|}
\hline Causal Belief & $\begin{array}{c}\mathrm{ID} \\
\mathrm{M}(\mathrm{SD})\end{array}$ & $\begin{array}{c}\text { Schizophrenia } \\
\text { M (SD) }\end{array}$ & $\mathrm{T}$ value & $\overline{\text { Cohen's d }}$ \\
\hline \multicolumn{5}{|l|}{ Biomedical } \\
\hline Virus/ brain infection & $3.23(1.78)$ & $3.87(1.86)$ & $-12.92 *$ & 0.35 \\
\hline Genetic factors & $3.86(1.78)$ & $4.31(1.81)$ & $-9.22 *$ & 0.25 \\
\hline Complications at birth & $3.49(1.74)$ & $3.14(1.79)$ & $7.10^{*}$ & 0.20 \\
\hline Brain abnormality & $4.04(1.85)$ & $4.72(1.78)$ & $-12.90 *$ & 0.37 \\
\hline Meningitis & $3.08(1.66)$ & $2.98(1.71)$ & 2.37 & 0.06 \\
\hline \multicolumn{5}{|l|}{ Adversity } \\
\hline Family arguments & $3.65(1.69)$ & $3.72(1.78)$ & -1.38 & 0.04 \\
\hline Financial worries & $3.14(1.75)$ & $3.30(1.81)$ & $-3.25^{*}$ & 0.09 \\
\hline Suffering abuse as a child & $3.76(1.75)$ & $3.98(1.81)$ & $-4.81 *$ & 0.12 \\
\hline Recent trauma & $3.65(1.77)$ & $4.26(1.83)$ & $-12.63 *$ & 0.34 \\
\hline $\begin{array}{r}\text { Recent death of relative or } \\
\text { close friend }\end{array}$ & $3.64(1.80)$ & $4.16(1.85)$ & $-11.33^{*}$ & 0.28 \\
\hline \multicolumn{5}{|l|}{ Environment } \\
\hline Overly spoilt as a child & $3.70(1.86)$ & $2.20(1.47)$ & $30.10 *$ & 0.89 \\
\hline Lack of daytime occupation & $4.29(1.79)$ & $3.60(1.88)$ & $13.61^{*}$ & 0.38 \\
\hline Very poor schooling & $4.51(1.67)$ & $2.36(1.54)$ & $45.18^{*}$ & 1.34 \\
\hline From single-parent family & $2.95(1.76)$ & $2.37(1.63)$ & $13.85^{*}$ & 0.34 \\
\hline Parents too lenient & $4.20(1.88)$ & $2.59(1.70)$ & $31.79 *$ & 0.90 \\
\hline $\begin{array}{l}\text { Lack of an intimate } \\
\text { relationship }\end{array}$ & $3.47(1.78)$ & $3.48(1.90)$ & -0.39 & 0.01 \\
\hline $\begin{array}{r}\text { Isolation from extended } \\
\text { family }\end{array}$ & $2.79(1.63)$ & $2.89(1.79)$ & -2.37 & 0.06 \\
\hline \multicolumn{5}{|l|}{ Supernatural } \\
\hline $\begin{array}{r}\text { Punishment for own past } \\
\text { wrongdoings }\end{array}$ & $2.54(1.71)$ & $2.48(1.84)$ & 1.40 & 0.03 \\
\hline $\begin{array}{r}\text { Strong religious or spiritual } \\
\text { beliefs }\end{array}$ & $1.99(1.44)$ & $2.26(1.71)$ & $-6.52 *$ & 0.17 \\
\hline Spirit possession & $1.65(1.34)$ & $1.92(1.61)$ & $-8.10^{*}$ & 0.18 \\
\hline $\begin{array}{r}\text { Punishment for parents' } \\
\text { wrongdoings }\end{array}$ & $2.46(1.67)$ & $2.29(1.71)$ & $4.75^{*}$ & 0.10 \\
\hline A test from God/ Allah & $1.81(1.56)$ & $1.82(1.59)$ & -0.36 & 0.01 \\
\hline
\end{tabular}


Table 3. Endorsement of causal beliefs by explanation given for intellectual disability vignette: Subscale means (standard deviations) and ANOVA results

\begin{tabular}{rccccc}
\hline & Total & $\begin{array}{c}\text { Intellectual } \\
\text { Disability } \\
(\mathrm{n}=490)\end{array}$ & $\begin{array}{c}\text { Specific LD/ } \\
\text { ASD } \\
\mathrm{n}(65)\end{array}$ & $\begin{array}{c}\text { Other } \\
\text { Explanation } \\
(\mathrm{n}=1142)\end{array}$ & F value \\
\hline $\begin{array}{r}\text { Causal Beliefs } \\
\text { Biomedical }\end{array}$ & $3.55(1.38)$ & $4.28(1.19)$ & $4.02(1.25)$ & $3.21(1.36)$ & $122.66^{*}$ \\
Adversity & $3.60(1.32)$ & $3.39(1.32)$ & $3.16(1.17)$ & $3.71(1.31)$ & $14.23^{*}$ \\
& & & & & \\
Environment & $3.71(1.17)$ & $3.09(1.16)$ & $3.03(1.15)$ & $4.01(1.04)$ & $136.68^{*}$ \\
Supernatural & $2.09(1.11)$ & $1.72(0.88)$ & $1.81(0.99)$ & $2.26(1.16)$ & $50.65^{*}$ \\
\hline$* p<0.001$ & & & & &
\end{tabular}


Table 4. Endorsement of different causal beliefs by explanation given for schizophrenia vignette: Subscale means (standard deviations) and ANOVA results

\begin{tabular}{ccccccc}
\hline & Total & $\begin{array}{c}\text { Schiz./ } \\
\text { Psychosis } \\
(\mathrm{n}=365)\end{array}$ & $\begin{array}{c}\text { Depression } \\
(\mathrm{n}=1527)\end{array}$ & $\begin{array}{c}\text { Mental Illness } \\
(\text { general/ other }) \\
(\mathrm{n}=470)\end{array}$ & $\begin{array}{c}\text { Other } \\
\text { Explanation } \\
(\mathrm{n}=308)\end{array}$ & F value \\
\hline $\begin{array}{c}\text { Causal Beliefs } \\
\text { Biomedical }\end{array}$ & $3.81(1.32)$ & $4.20(1.20)$ & $3.38(1.30)$ & $3.97(1.29)$ & $3.35(1.34)$ & $34.26^{*}$ \\
Adversity & $3.85(1.39)$ & $3.59(1.34)$ & $4.25(1.37)$ & $3.82(1.34)$ & $3.97(1.45)$ & $10.60^{*}$ \\
& & & & & & \\
Environment & $2.73(1.22)$ & $2.18(1.03)$ & $3.15(1.18)$ & $2.60(1.11)$ & $3.32(1.24)$ & $67.01^{*}$ \\
Supernatural & $2.13(1.28)$ & $1.59(0.87)$ & $2.30(1.34)$ & $1.98(1.10)$ & $2.91(1.52)$ & $66.63^{*}$ \\
\hline & & & & & &
\end{tabular}


Table 5. Correlations between individual causal items and social distance

\begin{tabular}{|c|c|c|}
\hline \multirow[t]{2}{*}{ Causal Item } & \multicolumn{2}{|c|}{ Social Distance } \\
\hline & $\begin{array}{l}\text { Intellectual } \\
\text { Disability }\end{array}$ & Schizophrenia \\
\hline \multicolumn{3}{|l|}{ Biomedical } \\
\hline Virus/ brain infection & $-0.11 *$ & $0.08^{*}$ \\
\hline Genetic factors & $-0.17^{*}$ & -0.03 \\
\hline Complications at time of birth & $-0.18^{*}$ & 0.01 \\
\hline Brain abnormality & $-0.14 *$ & $0.08^{*}$ \\
\hline Meningitis & $-0.14^{*}$ & 0.03 \\
\hline \multicolumn{3}{|l|}{ Adversity } \\
\hline Family arguments & 0.05 & -0.01 \\
\hline Financial worries & 0.00 & -0.04 \\
\hline Suffering abuse as a child & -0.04 & -0.03 \\
\hline Recent traumatic incident & $-0.10^{*}$ & -0.06 \\
\hline Recent death of relative or close friend & -0.04 & -0.06 \\
\hline \multicolumn{3}{|l|}{ Environment } \\
\hline Overly spoilt as a child & $0.26^{*}$ & 0.05 \\
\hline Lack of daytime occupation & $0.15 *$ & 0.03 \\
\hline Very poor schooling & 0.07 & 0.03 \\
\hline Being from a single-parent family & $0.13 *$ & 0.02 \\
\hline Parents too lenient & $0.30 *$ & $0.09 *$ \\
\hline Lack of an intimate relationship & 0.05 & 0.03 \\
\hline Isolation from extended family & 0.03 & -0.03 \\
\hline \multicolumn{3}{|l|}{ Supernatural } \\
\hline Punishment for own past wrongdoings & $0.08^{*}$ & $0.10^{*}$ \\
\hline Strong religious or spiritual beliefs & 0.06 & $0.08^{*}$ \\
\hline Spirit possession & $0.09 *$ & $0.12 *$ \\
\hline Punishment for parents' wrongdoings & $0.13 *$ & $0.10^{*}$ \\
\hline A test from God / Allah & 0.05 & $0.09 *$ \\
\hline
\end{tabular}

* Spearman's rho significant at $p<0.05$ (Bonferroni corrected) 
Table 6. Correlations between causal beliefs and social distance for intellectual disability and schizophrenia vignettes $(N=1752)$

\begin{tabular}{lcc}
\hline Causal Beliefs & \multicolumn{2}{c}{ Social Distance } \\
& Intellectual Disability & Schizophrenia \\
\hline Biomedical & $-0.19^{*}$ & 0.05 \\
Adversity & -0.04 & -0.07 \\
Environmental & $0.21^{*}$ & 0.05 \\
Supernatural & 0.12 & $0.14^{*}$
\end{tabular}

*Spearman's rho significant at *p<0.01 (Bonferroni corrected) 
Figure 1

\section{Causal Beliefs}

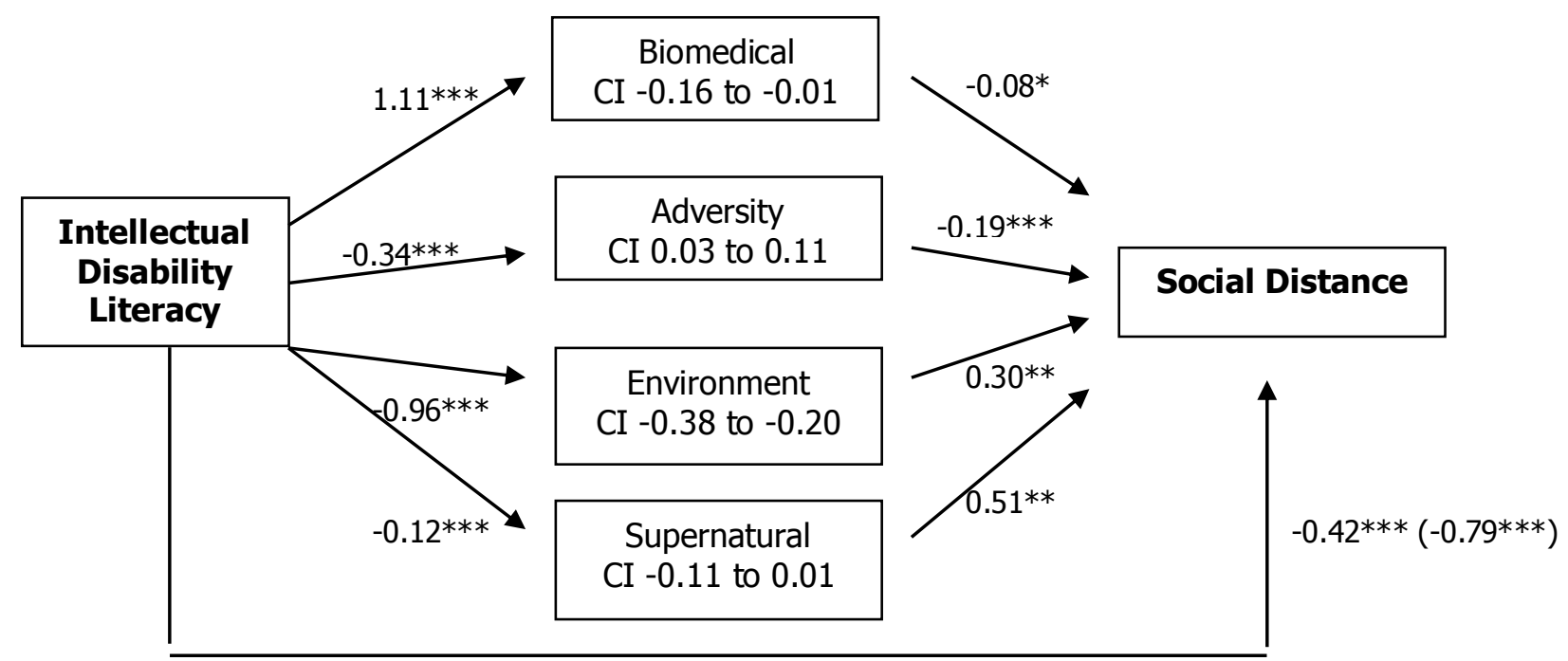

Figure 1. Associations between intellectual disability literacy, causal beliefs and social distance

Note: Confidence intervals (CI) reported are 95\% BCa bootstrap confidence intervals. Path values represent unstandardised regression coefficients. The value outside the parenthesis represents the total direct effect, from bootstrapping analyses, of recognition of intellectual disability on social distance after causal belief mediators were included. The value inside the parenthesis represents the total effect of recognition on social distance, prior to the inclusion of mediating variables. ${ }^{*} p>0.05,{ }^{*} p<0.01,{ }^{* * *} p<0.001, n=1572$. 
Figure 2

\section{Causal Beliefs}

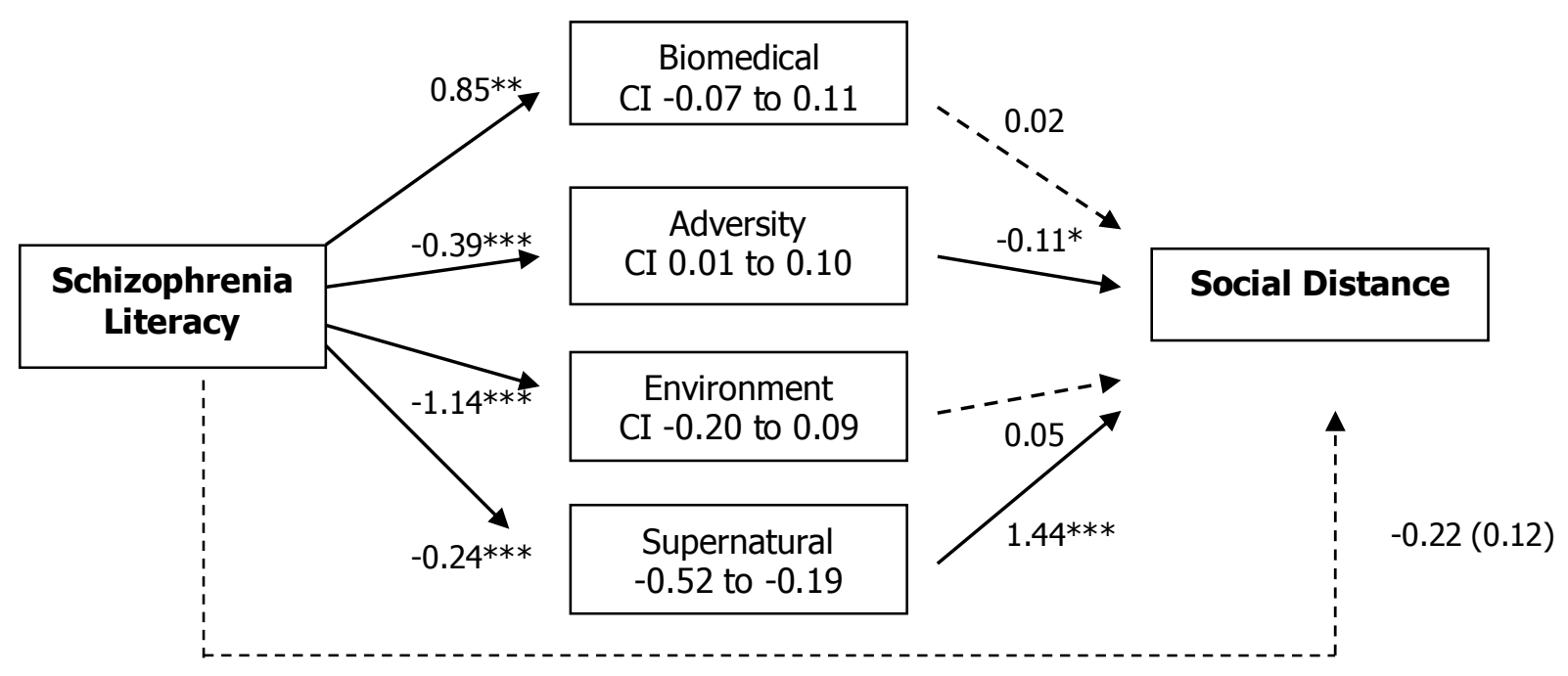

Figure 2. Associations between schizophrenia literacy, causal beliefs and social distance ${ }^{*} p>0.05, * * p<0.01, * * * p<0.001, n=668$ 